

\title{
Le quaternaire de la côte asturienne (Espagne)
}

\author{
Guy Mary, J. Medus, Georgette Delibrias
}

\section{To cite this version:}

Guy Mary, J. Medus, Georgette Delibrias. Le quaternaire de la côte asturienne (Espagne). Bulletin de l'Association française pour l'étude du quaternaire, 1975, 12 (1), pp.13-23. 10.3406/quate.1975.1253 . hal-03408236

\section{HAL Id: hal-03408236 \\ https://hal.science/hal-03408236}

Submitted on 29 Oct 2021

HAL is a multi-disciplinary open access archive for the deposit and dissemination of scientific research documents, whether they are published or not. The documents may come from teaching and research institutions in France or abroad, or from public or private research centers.
L'archive ouverte pluridisciplinaire HAL, est destinée au dépôt et à la diffusion de documents scientifiques de niveau recherche, publiés ou non, émanant des établissements d'enseignement et de recherche français ou étrangers, des laboratoires publics ou privés. 


\section{Le quaternaire de la côte asturienne (Espagne)}

\section{Guy Mary, J. Medus, Georgette Delibrias}

\section{Abstract}

Three sea levels are bordering the Asturian Coast between 5 and $40 \mathrm{~m}$. The 100/150 m and 165/250 m marine platforms date from the Tertiary. The differences in level are the result of a westward decreasing tectonic upthrust. Some marine oscillations took place during the Flandrian. A propensity to upthrust seems to subsist. The pollen contents of dated peats would indicate a phytodynamic delay during the Holocen with regard to the Basque country.

\section{Résumé}

Trois niveaux marins s'étagent entre $5 \mathrm{~m}$ et $40 \mathrm{~m}$ sur la côte asturienne. Les plateformes marines de 100/150 m et de 155/260 $\mathrm{m}$ datent du Tertiaire. Les dénivellations résultent d'un soulèvement tectonique décroissant vers l'Ouest. Des oscillations marines ont eu lieu pendant le Flandrien. Une tendance au soulèvement semble subsister. Le contenu pollinique de tourbes datées indiquerait un retard phytodynamique au cours de l'Holocène par rapport au Pays Basque.

Citer ce document / Cite this document :

Mary Guy, Medus J., Delibrias Georgette. Le quaternaire de la côte asturienne (Espagne). In: Bulletin de I'Association française pour l'étude du quaternaire, vol. 12, n¹, 1975. pp. 13-23;

doi : https://doi.org/10.3406/quate.1975.1253

https://www.persee.fr/doc/quate_0004-5500_1975_num_12_1_1253

Fichier pdf généré le 06/11/2020 


\title{
LE QUATERNAIRE DE LA COTE ASTURIENNE (ESPAGNE)* $\square$

\author{
par G. MARY ${ }^{* *}$,J. MEDUS ${ }^{* * *}$ et G. DELIBRIAS ${ }^{* * *}$
}

\begin{abstract}
RESUME
Trois niveaux marins s'étagent entre $5 \mathrm{~m}$ et $40 \mathrm{~m}$ sur la côte asturienne. Les plateformes marines de $100 / 150 \mathrm{~m}$ et de $155 / 260 \mathrm{~m}$ datent du Tertiaire. Les dénivellations résultent d'un soulèvement tectonique décroissant vers l'Ouest. Des oscillations marines ont eu lieu pendant le Flandrien. Une tendance au soulèvement semble subsister. Le contenu pollinique de tourbes datées indiquerait un retard phytodynamique au cours de l'Holocène par rapport au Pays Basque.
\end{abstract}

\begin{abstract}
Three sea levels are bordering the Asturian Coast between 5 and $40 \mathrm{~m}$. The $100 / 150 \mathrm{~m}$ and $165 / 250 \mathrm{~m}$ marine platforms date from the Tertiary. The differences in level are the result of a westward decreasing tectonic upthrust. Some marine oscillations took place during the Flandrian. A propensity to upthrust seems to subsist. The pollen contents of dated peats would indicate a phytodynamic delay during the Holocen with regard to the Basque country.
\end{abstract}

La côte septentrionale espagnole qui borde le Golfe de Gascogne est méconnue quant aux plages quaternaires. Guilcher (1955) identifia à la Franca un rivage de 6 à $7 \mathrm{~m}$. Les auteurs du livret guide de l'excursion $\mathrm{N}$ du $\mathrm{V}^{\mathrm{e}}$ Congrès de l'Inqua (1957) ont signalé quelques gisements. Grisez (1961) a trouvé un affleurement à $7 \mathrm{~m}$ près de Luarca et deux lambeaux fluviatiles à $27 \mathrm{~m}$. Les résultats exposés ici intéressent le segment médian de la côte cantabrique compris entre la ria de Ribadeo à l'Ouest et celle de la Rabia à l'Est. Ils ont trait aux niveaux marins quaternaires, à la remontée flandrienne, aux phénomènes morphoclimatiques et à la palynologie.

La côte s'allonge d'Ouest en Est marquée par quelques caps importants : Busto, Vidio, Vidrias et
Penas qui prend des allures de péninsule. De hautes falaises la forment. L'altitude de leur sommet atteint $100 \mathrm{~m}$ au cap de Penas et à la pointe de Vidrias. Elle décroît régulièrement vers l'Ouest. A Ribadeo, elle est d'environ $20 \mathrm{~m}$.

La chaine cantabrique s'étire parallèlement à la côte depuis les Pyrénées jusqu'en Galice. Sa surrection s'est produite au cours de l'ère tertiaire. Deux régions se distinguent au point de vue géologique. Le segment oriental appelé pyrénéo-cantabre (Feuillée, Rat 1971) appartient au domaine pyrénéen par la nature de ses terrains et par son évolution paléogéographique. Le segment occidental se compose pour l'essentiel des assises primaires de l'arc asturien retectonisées au Tertiaire. L'explora-

(*) Article déposé le 27 février 1975

(**) Laboratoire de Géologie - Faculté des Sciences, 72017 Le Mans Cedex.

(***) Laboratoire de Botanique historique et Palynologie - Equipe de Recherche Associée au C.N.R.S. $\mathrm{n}^{\circ} 404$ - Faculté des Sciences St Jérome, 13397 Marseille Cedex 4.

(****) C.N.R.S. Centre des faibles radio-activités, 91190 Gif-sur-Yvette. 




tion relative au Quaternaire a porté sur la côte de ce dernier segment depuis sa jonction avec le pays pyrénéo-cantabre qui se fait à San Vicente de la Barquera.

\section{A. - LES NIVEAUX MARINS}

\section{I - Les aplanissements còtiers}

Des surfaces planes comprises entre la mer et les chaînons montagneux caractérisent la morphologie de la bordure asturienne. Elles sont appelées "rasas" quand elles forment un palier au sommet des falaises et sont dominées par un versant. Elles sont dénommées "sierras planas" ou "Ilanos" quand il s'agit de plateaux. Tous les auteurs s'accordent pour reconnaître une plateforme apparemment unique à l'Ouest du Nalon et plusieurs gradins à l'Est. Deux origines ont été envisagées. Cueto y Rui Diaz (1930) puis Hernandez Pacheco E. (1932) ont pensé à un piémont continental. Pour Barrois (1882), les surfaces planes sont marines et dateraient du Miocène. Hernandez Sampelayo (1913) distingue deux niveaux marins dressés durant le Tertiaire. Llarena et Royo (1927) ont inventorié autant de plages soulevées que dans la synthèse de Deperet pour la Méditerranée. Birot et Sole Sabaris (1954) ont avancé une hypothèse tyrrhénienne pour la surface de l'Ouest asturien, c'est-à-dire là où elle est la plus basse. Llopis Llado (1955-1964) l'étendit à l'ensemble du palier même aux endroits où il est particulièrement élevé, comme aux caps de Busto et Vidio $(70$ à $80 \mathrm{~m})$. En raison de ces diverses idées, l'exposé fait le point sur la connaissance actuelle des aplanissements côtiers.

1) Le segment occidental. La plateforme tronque les couches cambro-ordoviciennes gréseuses puis schisteuses en particulier à Luarca et Navia. Son contact avec le versant du Sud se situe à $120 \mathrm{~m}$ près de Muros de Nalon, à $105 \mathrm{~m}$ à Otur au kilomètre 319,1 de la route nationale 634 et s'abaisse dès le voisinage de la ria de Ribadeo. Il est de l'ordre de $55 \mathrm{~m}$ au sud de cette ville. De nombreux dépôts de galets arrondis parsèment la surface. Les études morphoscopiques et morphométriques réalisées par Grisez (1961), Hernandez Pacheco F. et Asensio Amor (1959 à 1964) et par Mary (1967-1968-1969) conduisent à la certitude d'une action marine dans le façonnement des galets. Hernandez Pacheco F. et Asensio Amor (1961), partisans de l'hypothèse de l'origine continentale pensent à un remaniement marin de la couverture continentale. En effet, une formation alluviale de piémont existe entre les caps de Busto et de Vidio. Le plan de discordance avec le socle primaire montre des poches de galets très arrondis d'origine marine. Cette superposition prouve que la surface résulte d'abord de l'abrasion marine. Ceci renverse la succession des phénomènes avancée par Hernandez Pacheco F. et Asensio Amor.

A Salamir et à Las Hervedosas de Otur (Mary 1967) deux dépôts de galets existent à $175 \mathrm{~m}$ et $165 \mathrm{~m}$ d'altitude, soit $60 \mathrm{~m}$ au-dessus de la plateforme dite de $105 \mathrm{~m}$.

Deux niveaux marins s'étagent entre la gare de Ribadeo et la Ermita de Santa Cruz à $55 \mathrm{~m}$ environ et $100 \mathrm{~m}$ alors que sur la rive orientale de la ria ils déterminent un seul plan incliné.

En conclusion, le segment occidental qui paraît composé d'une seule surface comprend trois hauts niveaux marins à $175 / 165 \mathrm{~m}, 105 \mathrm{~m}$ et $60 \mathrm{~m}$.

\section{2) Le segment oriental}

Du rio Nalon à Aviles, une haute surface atteint $225 \mathrm{~m}$. Des galets très arrondis, puis des sables et enfin des argiles à lentilles de galets et de sables y affleurent d'amont en aval. La disposition des sédiments s'apparente à celle d'un dépôt deltaïque. Des lignites trouvés à la base ont livré une palynoflore d'âge sparnacien (Mary - Medus, 1971). L'autre palier se situe en contrebas à $135 \mathrm{~m}$.

Dans la péninsule du Penas, deux gradins s'identifient également au Monte Areo. L'inférieur s'élève jusqu'à $180 \mathrm{~m}$, le plus haut atteint $260 \mathrm{~m}$. Les deux recèlent des dépôts de galets marins, notamment le plus bas près du phare de Penas (Mary, 1970) et du Cabo Torres (Virgili et al 1968). Des indices topographiques conduisent à penser que la partie située en aval pourrait constituer un troisième niveau de $120 \mathrm{~m}$.

Entre Gijon et Berbes, une seule surface arase le jurassique gréseux et conglomératique.

De Berbes à San Vicente de la Barquera, le substratum est composé par des écailles de grès d'âge skiddavien pour l'essentiel chevauchant les calcaires dinantiens. Sur les grès, deux magnifiques surfaces planes sont conservées, l'une à $260 \mathrm{~m}$, 


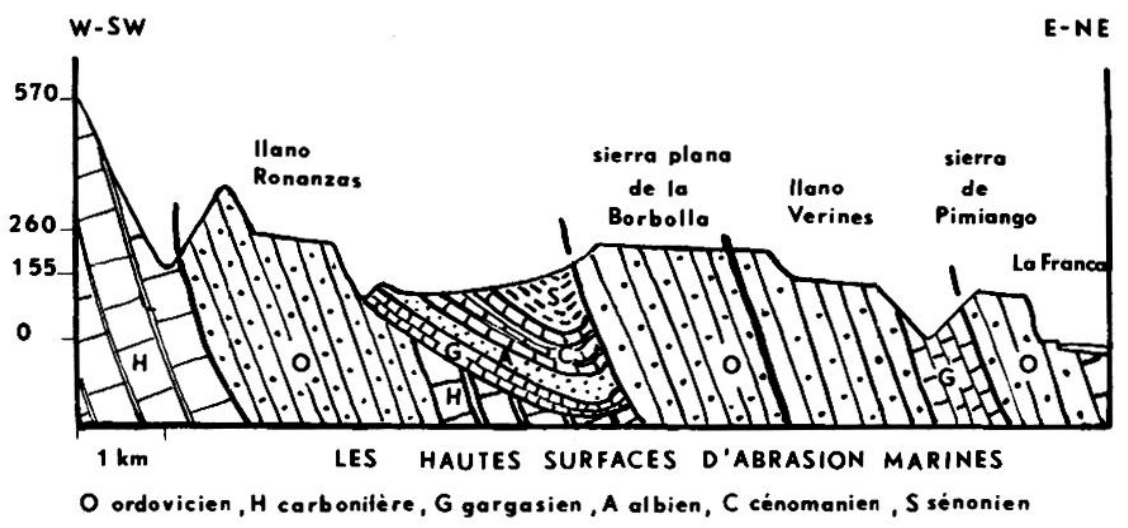

l'autre à $155 \mathrm{~m}$. Elles portent de nombreux placages de galets dont l'origine marine est indéniable. En contrebas, existe une banquette et des couloirs d'altitude variable taillés dans les calcaires dinantiens très karstifiés. Ces aplanissements ne montrent pas de dépôts marins. Leur aspect actuel résulte de l'évolution karstique des surfaces précédentes.

\section{3) Résumé}

A l'exception de celle qui tronque les calcaires dinantiens, les surfaces sont le fait de l'abrasion marine. Comme les paliers d'un compartiment ne se retrouvent pas dans le voisin, force est d'admettre l'existence de deux hautes surfaces que des manifestations de tectonique cassante ont fragmentées. Les morceaux ont été décalés les uns par rapport aux autres après chaque nivellement. Aux systèmes de $260-165 \mathrm{~m}$ et de $155 / 105 \mathrm{~m}$ s'ajoute le niveau de 50-60 m près de la ria de Ribadeo, et qui pourrait atteindre $120 \mathrm{~m}$ au Cap de Penas.

Seule la plus haute surface a fourni des documents paléontologiques qui la datent du Sparnancien près des Aviles. Mais dans l'Est des Asturies, elle est postérieure au Nummulitique qu'elle arase aussi. Cependant la présence de klippes sédimentaires et de décharges détritiques grossières dans l'Eocène supérieur et l'Oligocène de la plage de San Vicente de la Barquera permet de penser que les mouvements tectoniques ont été continus au cours du Paléogène dans ce secteur du domaine pyrénéo-cantabre tandis que la partie occidentale du pays asturien demeurait relativement plus stable de telle sorte que la plus haute surface d'abrasion y apparaît comme ayant eu une longue histoire complexe depuis le début de l'Eocène jusqu'au Miocène. Mais celle de 155/105 m n'a fourni aucun fossile et son âge demeure indéterminé entre le Miocène et le Quaternaire. Toutefois des niveaux marins plus bas qu'elle restreignent l'incertitude.

\section{II - Les niveaux marins quaternaires}

Des dépôts marins apparaissent à diverses altitudes entre la mer et les hautes plateformes d'abrasion. Deux tronçons côtiers en possèdent : celui de Berbès - San Vicente de la Barquera et celui de l'Ouest asturien.

\section{1) Niveaux du tronçon Berbès - San Vicente de la Barquera}

A l'est de la station balnéaire de La Franca, la mer démolit une surface de $5,5 \mathrm{~m}$ à $6 \mathrm{~m}$ taillée dans les grès skiddaviens. D'innombrables îlots en demeurent de même qu'une banquette adossée à une falaise ancienne. La vielle encoche se voit en trois points à l'extrémité orientale du gisement. Sur les longues apophyses, des graviers et du sable revêtent le platier

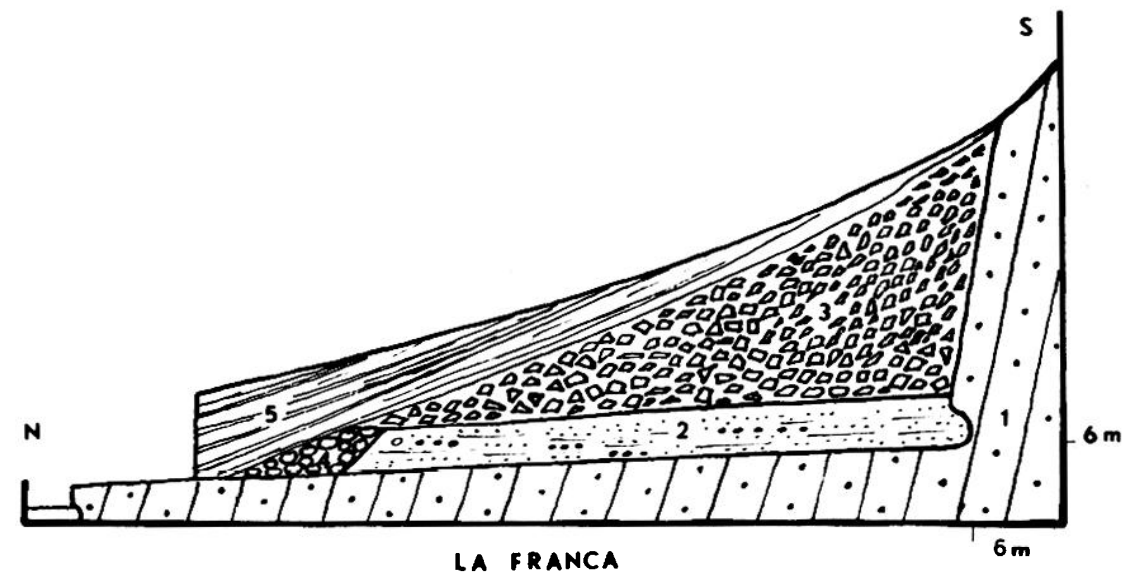

1 grès ordoviciens, 2 premier dépôt morin, 3 éboulis, 4 secand dépót morin , 5 paléosols 




sur une épaisseur de $1,50 \mathrm{~m}$. Cette formation est brusquement interrompue à l'aval par un cordon de petits blocs et de gros galets dont certains sont à peine émoussés. Puis le platier devient nu. Un éboulis enfouit le tout.

La phase sédimentaire relativement fine de l'amont indique que le dressage du platier était achevé et que la falaise avait atteint un profil en équilibre avec le niveau marin de 5,5 à $6 \mathrm{~m}$. La construction du cordon de l'aval est postérieure et signifie soit un stade de retrait soit plus vraisemblablement une réinvasion marine après un recul car le premier dépôt a été éliminé en aval. Les matériaux grossiers et en particulier ceux non émoussés ne peuvent provenir que de l'éboulis qui s'était progradé pendant le retrait de la mer, et qui a repris son extension après l'édification du cordon. Ainsi sur le même platier de $5,5 \mathrm{~m}$ à $6 \mathrm{~m}$ se sont déroulés deux ingressions marines.

Le front de l'éboulis qui déborde le cordon montre des paléosols tourbeux et à peu de distance une tourbe épaisse de $1 \mathrm{~m}$ repose directement sur le substratum. Le paléosol inférieur et la tourbe datent de plus de 35000 ans BP. (Gif 2707 et Gif 3052) Leurs spectres polliniques sont si semblables qu'ils ne peuvent être que contemporains. Les pourcentages relatifs des A.P. varient entre $80-90 \%$. Les taxons Betula et Alnus dominent. Leurs courbes sont d'ailleurs complementaires. Quand la proportion de l'aulne croît les taux de Ilex aquifolium, de Corylus et secondairement de Quercus augmentent. Quercus ilex, Ulmus et Carpinus accompagnent ces thermophiles. Cette association peut correspondre à un interstade würmien soit celui d'Hengelo soit un quelconque des plus anciens, ou même à une phase de l'Eemien. Comme les données sur la végétation würmienne en Espagne sont pratiquement inexistantes (Nonn 1966) et que celles de France sont encore fragmentaires (Van Campo 1969), la situation chronologique des tourbes de La Franca n'est pas facile à établir. Cependant, peu de phases du Würm de Dordogne présentent des taux d'A.P. comparables (Leroi Gourhan A. 1969, Paquereau 1969-1970). Lorsqu'il en est ainsi, la proportion élevée des A.P. résulte soit d'un développement de la chênaie mixte, soit d'une augmentation du nombre des pins. Or, l'extension géographique de ces phénomènes a été démontrée (Bastin 1970, Bordes et al 1972). De ce fait, l'interprétation du diagramme pollinique des tourbes de La Franca ne peut être proposée en l'absence d'un plus grand nombre de données palynologiques régionales.

Dans le cas où l'interstade relatif aux tourbes serait celui d'Hengelo ou l'un des plus anciens, l'édification du cordon qui date d'une oscillation antérieure pourrait représenter une remontée des eaux du début du Würm et le premier dépôt marin de l'amont encore plus ancien indiquerait alors l'interglaciaire RissWürm. La progradation des éboulis aurait commencé avec le würm naissant puis aurait débordé le cordon postérieurement. 
D'autres traces du niveau de $5,50 \mathrm{~m} / 6 \mathrm{~m}$ existent tant sur les grès (San Antonin de Bedon, rive gauche de la ria de Tina menor) que sur les calcaires dinantiens où les dépôts sont conservés dans des grottes (Mary 1971). La position altimétrique pour le tronçon non déformé de Berbès - San Vicente de la Barquera permet seule l'assimilation à un même niveau. Les fossiles manquent et les rares rencontrés sont inclus dans la chappe de calcaire karstique qui agglomère les galets des cavernes. Il s'agit de débris de patelles et de gibbules.

Tronquant les couches d'âge Eocène supérieur et Oligocène des plages de San Vicente de la Barquera et de Jerra, deux paliers apparaissent à $5,50 / 6 \mathrm{~m}$ et $15 \mathrm{~m}$ environ. (Mary 1971). Des placages de galets et de sables, moins nets, se rencontrent vers $40 \mathrm{~m}$ d'altitude près du hameau de La Venta.

\section{2) Tronçon des Asturies occidentales}

Les sites sont plus rares sur les strates cambroordovociennes. Un bas niveau existe à la plage de Otur dans une grotte creusée dans les schistes llandeiliens. La base se trouve à $2,40 \mathrm{~m}$ au-dessus des hautes mers et des placages de galets se voient $4 \mathrm{~m}$ plus haut (Mary 1967). La plage de Porcia possède des dépôts à $5-6 \mathrm{~m}$ et à $15 \mathrm{~m}$. Enfin dans l'anse où se jette le rio Esba, trois anciens rivages s'étagent à 5-10 m, 16-19 m et 24-30 m (Mary 1968).

\section{3) Conclusion}

En se référant à la haute surface d'abrasion marine de 155-105 m, le tronçon Berbes - San Vicente de la Barquera a été plus soulevé que celui des Asturies occidentales. Trois bas niveaux marins se retrouvent le long de chacun des deux segments. Cette similitude suggère une identité d'âge entre ceux occupant la même position ordinale. Les différences altimétriques entre les dépôts se correspondant ne paraissent pas suffisamment significatives pour ceux dits de $6 \mathrm{~m}$ et de $15 \mathrm{~m}$. Mais elles le deviennent davantage pour les traces supérieures situées entre 26 et $29 \mathrm{~m}$ à l'Ouest contre $40 \mathrm{~m}$ à l'Est. Ainsi le soulèvement différentiel qui s'est manifesté après le nivellement de $155-100 \mathrm{~m}$ se serait poursuivi dans le même sens après la réalisation du plus ancien des niveaux récents.

Le rivage de $6 \mathrm{~m}$ appartient selon une très forte présomption à l'interglaciaire Riss-Würm et les oscillations précoces du début du Würm l’ont modifié en partie. Ceux de $15 \mathrm{~m}$ et de $24 / 40 \mathrm{~m}$ pourraient être rapportées aux interglaciaires Mindel - Riss et GunzMindel. Quoi qu'il en soit, l'hypothèse d'un âge tyrrhénien pour l'ensemble de la plateforme de $155-105 \mathrm{~m}$ se trouve éliminée. Seules les parties les plus basses ont pu être retouchées à cette époque.

\section{III - Les dépôts marins inférieurs à 6 m}

Plus bas que le gisement de $6 \mathrm{~m}$, des dépôts marins pourraient indiquer des variations récentes du niveau moyen de la mer sur la côte asturienne.

\section{1) Plages de la Jerra et de San Vicente de la Barquera}

Sur la plage de la Jerra, une formation tourbeuse à noisettes est immergée à marée haute (Jerra 3 ). Elle affleure à $25 \mathrm{~m}$ de la falaise. Des restes de troncs et de racines se voient. L'âge trouvé par radiocarbone s'établit à $5250 \pm 90 \mathrm{BP}$ (Gif 1893). Le vallon d'oyambre qui débouche en face montre une autre tourbe (Jerra 1) qui tapisse le fond de la branche orientale du talweg. Les niveaux 5 et $25 \mathrm{~cm}$ contiennent des restes planctoniques marins (acritarches). Ils indiquent de brèves incursions marines alors que la tourbière se développait. Son âge est 5880 ans \pm 130 BP (Gif 2635). La branche occidentale recèle aussi une tourbe (Jerra 2) datée de 5300 ans \pm 120 BP (Gif 2917). Son installation signifie que les eaux marines étaient retirées et que le maximum de l'invasion a pu se produire entre la disparition de la tourbière la plus âgée et la formation de la seconde. Comme les datations portent sur le lit toubeux en contact avec le substratum marneux du tertiaire, la différence d'âge entre Jerra 2 et Jerra 3 , semble dénoter une conquête progressive de la plage par la flore continentale, à la suite du reflux marin. Depuis, la mer a réenvahi la partie exondée.

Près de l'embouchure de l'arroyo de Bederna sur la plage voisine de San Vicente de la Barquera un lit de galets marins épais de $60 \mathrm{~cm}$, repose sur les marnes tertiaires à $80 \mathrm{~cm}$ au-dessus du haut de l'estran sableaux. Une formation tourbeuse puissante de $60 \mathrm{~cm}$ interrompt le cordon de galets et s'incline de $10^{\circ}$ vers l'exe du talweg. Au contact de l'horizon de galets, la tourbe s'est développée dedans. La datation a donné 4770 ans \pm 110 B.P. (Gif 2636). Antérieurement à ce moment, l'embouchure de l'arroyo de Bederna a été colmatée par des galets à un niveau légèrement supérieur à l'actuel. Puis le ruisseau a incisé le dépôt et une tourbe s'est édifiée. Toutefois, il peut s'agir d'un simple colmatage par régularisation du trait de côte tout autant que d'une oscillation.

Tels que les faits apparaissent sur la plage de la Jerra, leur interprétation va dans le sens d'une oscillation du niveau marin au cours de la phase climatique appelée Atlantique. Si les dépôts de l'arroyo de Bederna correspondent à une variation du niveau de la mer, elle se distinguerait de la précédente car la tourbière de la Jerra était arrêtée alors que celle de la plage de San Vicente de la Barquera fonctionnait, un millénaire plus tard. Ces deux estrans voisins auraient alors enregistré deux oscillations qui s'apparenteraient à celles de la courbe de R.W. Fairbridge (1961) et à celles détectées par M. Ters sur les côtes françaises (1973).

\section{2) Asturies occidentales et centrales}

Des traces possibles de dépôts marins dominant de 1 à $3 \mathrm{~m}$ les hautes mers subsistent à l'embouchure de nombreux vallons dans les Asturies occidentales. Elles se présentent toujours sous la forme d'un lit de galets dont l'épaisseur varie de 30 à $50 \mathrm{~cm}$. Il occupe le fond du talweg à son arrivée sur la plage et ne se prolonge 
pas en amont. Il repose souvent sur des éboulis ordonnés, indurés, parfois ferruginisés. Fréquemment un sol puissant d'une vingtaine de centimètres le recouvre. Ces gisements se voient en particulier à la plage sise près de Barcia, à celles de Campiello, de Portizuelo, de Molinos, etc. Le fait ne paraît pas accidentel pour l'attribuer systématiquement à des tempêtes. De plus, la couche arable lui confère une certaine ancienneté. A Las Fontias, un gisement plus pauvre en galets recèle une faune de Patella vulgata, de Gibbula lineata et de Purpura hoemastoma dont l'âge est $1920 \pm 110 \mathrm{BP}$ (Gif 1267). Les dépôts signalés ici sont groupés en un même niveau à cause de leur position altimétrique, mais ils peuvent appartenir à des oscillations distinctes. S'ils n'établissent pas la certitude d'un rivage un peu plus haut que l'actuel au début de l'ére chrétienne, ils en suggèrent la probabilité.

La péninsule de Penas, possède trois sites plus nettement démonstratifs.

A la plage de Tenrero (San Cristobal de Verdicio), une couche de galets gît sur l'argile de décalcification des calacires dévoniens qui apparaissent une trentaine de centimètres plus bas. Du sable marin consolidé puis meuble la surmonte. La puissance complète du dépôt est de l'ordre de $0,75 \mathrm{~m}$. Il domine le sommet de la plage de $0,30 \mathrm{~m}$ au plus bas et s'élève suivant la falaise jusqu'à $2,50 \mathrm{~m}$. A cette hauteur, une rupture de pente apparraît. Elle se poursuit dans le pré concentriquement à la baie actuelle. Un système dunaire la cache en partie. En ce lieu, un niveau marin légèrement supérieur à l'actuel a non seulement laissé des dépôts mais il s'est inscrit géomorphologiquement.

Sur la rive gauche de l'ensenada de Banugues, entre le fond de la baie et de l'ancien cimetière, une couche de $1 \mathrm{~m}$ de sable marin enrichi en lutites gît sur les argiles de décalcification du calcaire dévonien, épaisses de 1, à $1,50 \mathrm{~m}$. Des galets et de rares débris de gastropodes marins y sont dispersés. La formation domine la plage actuelle de $2,50 \mathrm{~m}$ au maximum.

Une coupe sensiblement identique se voit à Xivares. La datation de coquilles de Purpura hoemastoma a donné $2150 \pm 110$ ans BP (Gif 897) (Mary, 1968). Ce dernier site indique que 150 ans avant l'ère chrétienne la mer atteignait une limite supérieure à l'actuelle. Le dépôt de Las Fontias pourrait signifier un épisode distinct du précédent comme y appartenir.

\section{3) Signification des dépôts marins inférieurs à $6 \mathrm{~m}$.}

Globalement ils démontrent des oscillations récentes du niveau de la mer. En l'absence de datation les corrélations entre les sites et leur chronologie relative ne peuvent être convenablement établies. Mais les sommets se situent toujours au-dessus du niveau actuel alors que pour la côte atlantique française Ters (1973) les constate plutôt au-dessous. Faut-il voir dans cette différence de comportement l'indice d'une manifestation de la poursuite de l'orogenèse pyrénéocantabre.

\section{B. - LES EVENEMENTS MORPHOCLIMATIQUES QUATERNAIRES}

Parmi toutes les "plages soulevées" du niveau de $6 \mathrm{~m}$ et attribuables à l'interglaciaire Riss-Würm, seule celle de La Franca possède une couverture de pierraille anguleuse, dont la signification au premier abord n'est pas convaincante en raison de la présence d'une haute falaise de grès skiddaviens. Les éboulis ont $\mathrm{pu}$ se produire pour toute autre cause que le froid, ne serait-ce que la verticalité. La mise en place était achevée avant le découpage de la plateforme par la remontée flandrienne. Elle coincide avec des épisodes reconnus froids en d'autres régions. Si auprès de la falaise fossile la disposition des cailloux rappelle plutôt celle d'éboulements et d'éboulis, en s'éloignant elle s'ordonne et montre une alternance de lits grossiers et d'horizons sableux se terminant par des paléosols. Les éboulis ordonnés peuvent désigner un climat périglaciaire. En conclusion, le revêtement de cailloux anguleux de La Franca est très certainement un head comme l'avait dit Guilcher (1955) et son âge est würmien.

Le cap Esbarradoiro fournit un autre exemple de head (Mary, 1971). Situé à l'ouest de Luarca, ce cap coïncide avec la fermeture de l'anticlinal de Esbarradoiro moulé dans les grès armoricains qui comportent quelques assises schisteuses. L'axe est creusé d'un cirque s'ouvrant sur la mer. Sur le flanc occidental se superposent des coulées faites d'une matrice argileuse à fins ćléments de schistes emballant de la pierraille et des blocs de grès et de schistes. La coulée inférieure descend sous la mer. Une niche creusée $6 \mathrm{~m}$ au-dessus des hautes mers contient des galets arrondis agglomérés par un ciment ferrugineux. La mise en place du head est antérieure à l'établissement du niveau de $6 \mathrm{~m}$ et elle date soit du Riss, soit du début du Würm.

Les vallons des Asturies occidentales déjà signalées pour recéler un niveau marin probable de 2 à $3 \mathrm{~m}$ contiennent également des éboulis, de même que d'autres suspendus très au-dessus de l'estran. Ainsi la vallée de Santa Ana arrive $40 \mathrm{~m}$ au-dessus de la grève. Un dépôt épais de $4 \mathrm{~m}$ en tapisse le fond. Il débute par une assise de blocaille sans matrice et dont les éléments sont disposés à plat parallèlement aux versants. Par-dessus reposent $60 \mathrm{~cm}$ d'une formation sablo-graveleuse à menus éclats de $20 \mathrm{~cm}$, une nouvelle couche de $60 \mathrm{~cm}$ d'éléments fins et un niveau terminal de $40 \mathrm{~cm}$ de blocaille. Les assises se correspondent d'un versant à l'autre et un peu en amont l'interfluve qui sépare les deux ruisseaux affluents porte les éboulis. Les cours d'eau ont depuis sa mise en place incisé le dépôt et le substratum sous-jacent. Les autres sites montrent de la même façon un éboulis ordonné. La fréquence d'une telle formation élimine l'hypothèse du phénomène accidentel et conduit à admettre une cause unique. La qualité et la disposition des terrains n'interviennent pas car les 
gisements existent aussi bien en pays gréseux qu'en région schisteuse, sur des strates verticales comme sur des assises horizontales. La disposition des éléments de l'éboulis dénote un apport latéral à partir des versants sans aucun remaniement longitudinal. Il s'agit de ruisseaux très courts nés sur la plateforme de $105 \mathrm{~m}$, à pente longitudinale forte, encaissés entre des versants très raides. La prédominance de l'apport latéral dans ces conditions topographiques favorables à une grande énergie semblerait alors correspondre à une réduction notable du ruissellement allant jusqu'à sa disparition. L'aridité pourrait être une conséquence du froid. Comme ces éboulis sont parfois surmontés par le niveau de 2 à $3 \mathrm{~m}$ et jamais par celui de $6 \mathrm{~m}$, la phase climatique contemporaine de leur formation se situe à la fin du Würm, probablement au cours du tardiglaciaire.

En résumé, la côte asturienne a probablement connu à basse altitude des épisodes climatiques froids. Cependant il faut noter la rareté des phénomènes périglaciaires, l'absence de feston de cryoturbation, de fente en coin. Les conditions périglaciaires paraissent globalement moins rigoureuses qu'en France.

\section{C. - LA PALYNOLOGIE}

Dans les chapitres précédents, des tourbes furent utilisées pour dater des oscillations du niveau marin. Mais elles apportent par leur contenu pollinique une connaissance plus précise des événements climatiques de la fin du Pléistocène et de l'Holocène que les phénomènes morphologiques. Elles sont ici étudiées de la plus ancienne à la plus récente. La tourbe de La Franca a été traitée précédemment.

\section{1) Grotte de Tina Mayor}

La grotte de la plage de "las arenas" près de l'embouchure de la ria de Tina Mayor est un témoin du niveau de $6 \mathrm{~m}$. Le remblaiement de galets marins est couronné par une couche d'argile de décalcification descendue par une cheminée. Le spectre sporollinique obtenu se compose de la façon suivante :

\begin{tabular}{|c|c|c|}
\hline Juniperus & 1 & Rhamnacées \\
\hline Abies & 1 & Ledum \\
\hline Pinus & 21 & Artemisia \\
\hline Quercus & 4 & Caryophyllacées \\
\hline Carpinus & 1 & Anthémidées \\
\hline Alnus & 2 & Cichoriées \\
\hline Corylus & 7 & Graminées \\
\hline Ilex aquifolium & 2 & \\
\hline Oléacées & 1 & \\
\hline Total des AP & 40 & Total des NAP \\
\hline Spores monolètes & 55 & Spores trilètes \\
\hline \multicolumn{3}{|l|}{ Total des } \\
\hline pollenospores & 114 & \\
\hline Cicatricoporites & 4 & \\
\hline Varia & 15 & \\
\hline
\end{tabular}

L'âge des débris d'os sous-jacents $(10000 \pm 600$ BP Gif 1892) fixe la limite inférieure de ce spectre au Pré boréal. Cependant sa composition qualitative et quantitative supporterait bien une comparaison avec la zone PI du Moura en Pays Basque (Oldfield 1964). La présence de Ilex aquifolium s'accorde avec les données d'Olfield (1c). La quantité de Pinus évoque plus particulièrement la base de la zone $P 1$ du Moura alors que la datation obtenue à Tina Mayor coïncide avec l'âge du sommet. Autrement dit, un léger décalage chronostratigraphique des phases phytogéographiques apparaît entre le Pays Basque et les Asturies.

Le remaniement de spores d'âge crétacé (Cicatricosisporites) dans un niveau de colmatage d'un karst affectant les calcaires dinantiens (Mary 1971) suppose un drainage de vestiges de formations créracées dont les affleurements sont voisins.

\section{2) Jerra 1 .}

Le lit de tourbe de la branche orientale du vallon de Oyambre est épais de $25 \mathrm{~cm}$. Il est daté de $5880 \pm 130$ ans BP (Gif 2635). Cet âge autorise un rapprochement étroit avec le spectre de l'extrême base de la zone P3 du Moura. (Oldfield 1964). Les éléments de ressemblance existent bien. A Jerra, le chêne domine dans la chênaie mixte comme au Moura, le noisetier atteint encore des pourcentages élevés mais il diminue comme dans la zone P3 et le pin tient encore une place assez importante parmi les A.P. Mais à la différence du Moura, l'aulne ne se manifeste qu'en faible quantité et seulement au sommet du niveau. L'extension de l'aulne pourrait ainsi être légèrement plus tardive sur le rivage cantabrique qu'en Pays Basque.

En dehors de ceux figurés dans le tableau, la tourbe de Jerra 1 a fourni les taxons suivants: Mercurialis 1, Gallium 3, Campanula 1, Ephedra 1, Urticacées 3, Rhamnacées 1, Oléacées 1.

\section{3) Jerra 2}

L'horizon tourbeux du diverticule occidental du vallon mesure $60 \mathrm{~cm}$ d'épaisseur. L'âge de la base est $5300 \pm 120$ ans BP (Gif 2917). Il implique la nécessité de rapporter ce dépôt à la limite des zones VII $a$ et VII $b$ du Moura et non pas à la partie supérieure de la zone $P 1$ comme le suggère le diagramme pollinique caractérisé par une variation importante du rapport AP/NAP de bas en haut. Il prouve un déboisement que l'absence de grains de céréales ne permet pas d'attribuer à une influence humaine. Sans exclure totalement cette cause, le développement de cette phase de lande à Ericacées peut constituer une suite logique à une période de dénudation causée par un mouvement transgressif de la mer. La tourbe de Jerra 1 en a enregistré des incursions, et l'amplitude maximale se situerait entre $5880 \mathrm{BP}$ et $5300 \mathrm{BP}$. Les fréquences relatives des Caryophyllacées de certains niveaux étayent cette 

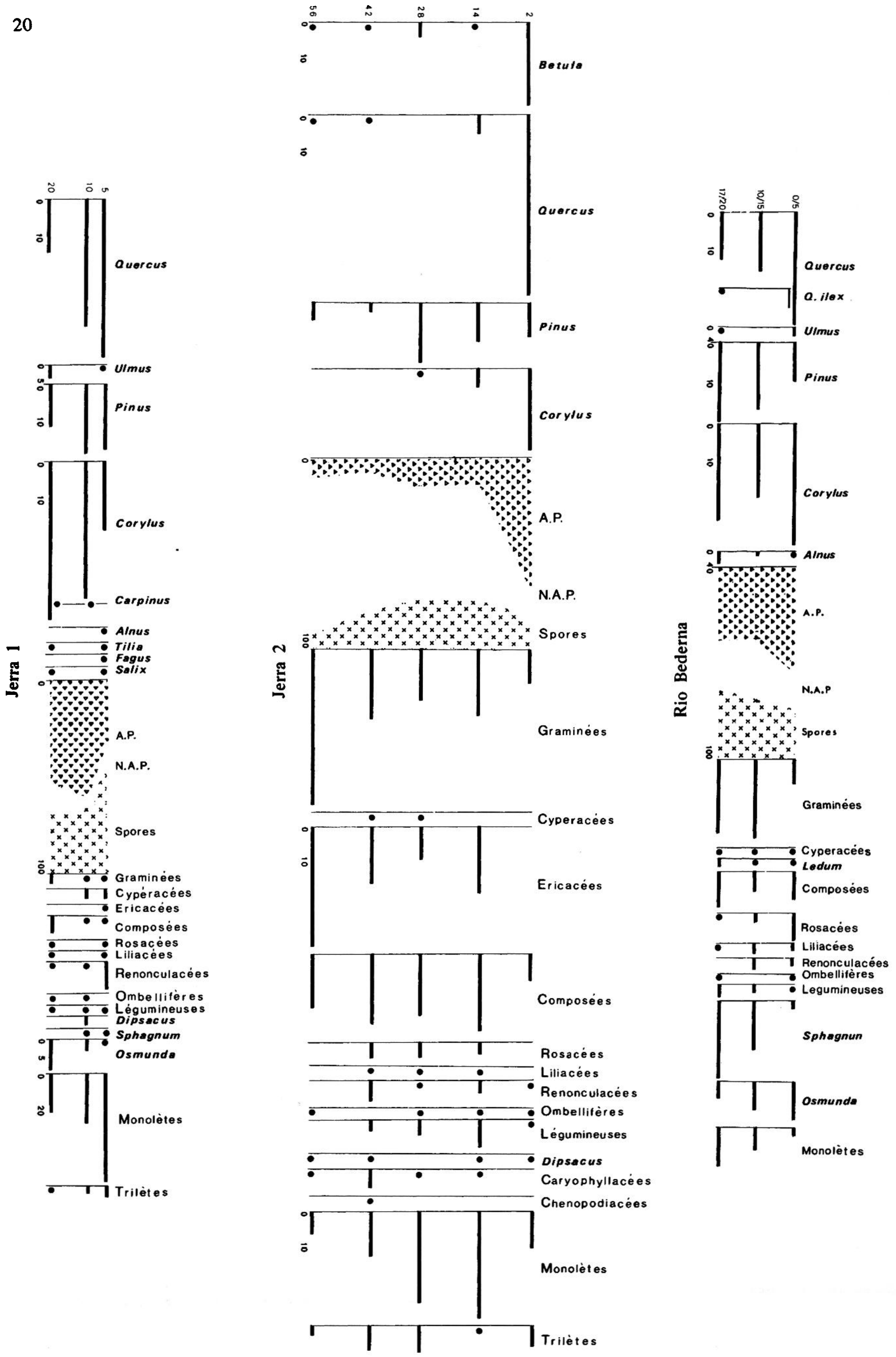
interprétation de même que l'augmentation brutale des AP, notamment de Quercus, au sommet. De plus cette explication liée à des processus dont les influences demeurent localisées au trait de côte pourrait également rendre compte de la lacune constatée par Oldfield (1964) dans les sédiments contemporains du Pays Basque.

\section{4) Rio Bederna}

Le lit tourbeux de ce gisement est daté de $4770 \pm 110$ ans BP (Gif 2636). Le diagramme palynologique montre que le chêne, le pin et le noisetier dominaient dans la couverture forestière. L'aulne et l'orme étaient très discrets. Dans le détail, la présence de Ledum palustre, à l'exception d'autres Ericacées, atteste, semble-t-il, une humidité que l'importance des sphaignes et les variations de l'aulne pourraient confirmer.

Il n'est guère possible de rapporter ces éléments à des faits similaires établis dans le voisinage. Au contraire, il faut noter la très faible représentation de l'aulne à Bederna par rapport à la place que ce taxon occupe en Pays Basque dans le gisement de Mouligna (Oldfield, 1960) alors que le laps de temps qui sépare ces deux sites est assez bref. Par ailleurs, au large de l'embouchure de la Gironde, Vigneaux (1973) met en évidence un épisode subboréal dans lequel Alnus paraît avoir une certaine importance. L'extrême réduction de l'aulne observée à Berderna autant que la place occupée par le pin et le noisetier dans le diagramme peuvent être le résultat de l'action humaine comme le suppose Oldfield 1960. Elle aurait eu pour effet d'accroître l'influence des modifications climatiques sur la végétation et aurait peut-être favorisé momentanément l'yeuse.

Dans les spectres de Bederna, cette déforestation ne se trouverait autrement marquée que par une sensible augmentation des herbacées (Graminées, Composées), les rudérales étant quasiment absentes. Le diagramme ne figure pas les taxons suivants: Caryophyllacées 1, Ephedra 1, Dipsacus 3, Lonicera 1, Urticacées 1, Polygala 1, Salix, Carpinus 2, Quercus suber 1, Oléacées 1.

\section{5) Discussion}

Le tableau ci-dessous réunit les datations actuellement connues des gisements de tourbes de l'Espagne atlantique. Les sites de Burela en Galice (Delibrias, Nonn et Van Gampo, 1964) et celui de Mougas (Nonn, 1966) ont été ajoutés pour compléter la séquence.

La documentation dont on dispose maintenant concerne surtout l'Atlantique et le Sub-Boréal, c'està-dire les zones VII a VII b de la chronostratigraphie anglaise (Godwin et Willis, 1959).

Un décalage des phases de végétation s'observe entre le Pays Basque et les Asturies. Il constitue une difficulté importante dans les tentatives de corrélation. L'essai d'une explication doit tenir compte de



son caractère constant ainsi que des comportements respectifs de Corylus et de Alnus.

Au Pays Basque comme dans les Asturies, Corylus occupe constamment dans les diagrammes du PostGlaciaire une place importante. A l'Atlantique, les pourcentages des éléments de la chênaie mixte autres que Quercus (Ulmus, Tilia) demeurent discrets (Oldfield, 1964, 162). Mais, le début de l'augmentation du taux de Alnus semble s'avérer plus tardif (postérieur à $4770 \pm 110 \mathrm{BP}$ ) dans les Asturies qu'en Pays Basque (5865 \pm 170 BP). Or MorzadecKerfourn (1969) signale la coïncidence de la "chute des pourcents de pollens d'aulne à la fin du SubBoréal. . . (avec) l'arrivée de la mer flandrienne". De plus le rôle de colonisateur que tient le noisetier lors des modifications de la végétation est bien connu. Ces deux facteurs peuvent rendre compte des particularités des spectres asturiens. En outre, comme les essences telles que Tilia, Fagus Ulmus, et Alnus, littéralement masquées dans les diagrammes par l'abondance de Corylys et, ou de Quercus, sont plus exigeantes du point de vue sol, les hypothèses d'un rajeunissement pédologique ou d'un retard de la pédogénèse doivent être envisagées. Plusieurs causes pourraient se trouver à l'origine de ces processus. Ainsi Nonn (1966) a supposé que de basses températures des eaux de surface, au large des côtes atlantiques de l'Espagne, au Finiglaciaire pouvaient fournir une interprétation à quelques spectres représentant une végétation de type exceptionnellement froid, pour l'époque à laquelle ils correspondaient. On pourrait penser que ces influences mésoclimatiques agissaient suffisamment pour ralentir l'évolution pédologique au moins au Dryas. Pour les phases du Post-Glaciaire, l'importance des mouvements eustatiques démontré par Godwin, Suggate et Willis (1968) doit être considérée. Ces mouvements dont certains témoins ont déjà été mis en évidence sur la côte des Asturies (Mary, 1971, 1972), sont inter- 
venus sur la distribution géomorphologique des gisements de tourbes du littoral asturien et breton (Morzadec-Kerfourn, 1969). Par conséquent ils ont pu, sur une aire limitée, être directement responsables de rajeunissements pédologiques, soit par abrasion soit par fossilisation des surfaces. A ces effets, il faudrait ajouter ceux, cumulatifs, d'une néotectonique (Mary, 1971) qui peuvent atteindre de grandes unités.

On ne saurait toutefois négliger un autre aspect de la question. Il s'agit des différences qui ont été signalées entre les corrélations palynologiques et les datations au radiocarbone. Celles-ci ont servi de base à l'établissement du tableau synoptique mettant en évidence le décalage chronostratigraphique. Mais on observe qu'il est fondé sur les datations fournies par deux gisements (Jerra 2 et Bederna) dont les spectres s'apparentent mieux à d'autres phases paléophytogéographiques que celles auxquelles les dates ont conduit. Et comme le suggèrent les variations de fréquences on pourrait rapporter le diagramme du gisement de Jerra 2 au Préboréal (zone IV-V) et celui de Bederna à l'Atlantique (zone VII a). Cependant la position stratigraphique des spectres de Jerra $I$ et de Tina Mayor coincide avec leur âge absolu respectif.

L'adoption d'un tel système permet de retrouver clairement les traits majeurs des fluctuations phytogéographiques post-glaciaires communs au sud de l'Angleterre, à la Bretagne et au Pays Basque et par conséquent il a l'avantage de présenter des résultats conformes à ceux obtenus par Oldfield (cf. notamment Quercus ilex dans Bederna).

Une accumulation des données permettra peutêtre ultérieurement une démonstration (et la coordination) de ces hypothèses; alors que les investigations actuelles n'autorisent pas encore de faire la part de chacune d'elle dans l'interprétation des particularités des modifications phytogéographiques des Asturies.

\section{BIBLIOGRAPHIE}

Barrois C, 1882. - Recherches sur les terrains anciens des Asturies et de la Galice. Mém. Soc. Géol. Nord, t. II.

Bastin B., 1970. - La chronostratigraphie du Würm en Belgique, à la lumière de la palynologie des loess et limons. Ann. Soc. Geol. Belgique, 93 (3) 545-580.

Birot P. et Sole Sabaris L., 1954. - Recherches morphologiques dans le Nord-Ouest de la Péninsule Ibérique. Mém. et Doc., C.N.R.S., t. IV, p. 7-61.
Bordes F., Laville H., Lumley H. de, Miskovsky J.C., Paquereau M.M., Pillard B., Prat F. et Renault-Miskovsky J., 1972. - Le Würmien II Tentative de corrélations entre le Languedoc méditerranéen (l'Hortus) et le Périgord (CombeGrenal). Etudes Quaternaires. Mém. $n^{\circ} 1$ : 353362.

Cueto y Rui Diaz E., 1930. - Nota acerca de las llanuras, rasas y sierras planas de la costa de Asturias. Bol. Real. Soc. Esp. de Hist. Nat., p. 241-254.

Delibrias G., Nonn H. et Van Campo M., 1964. Age et flore d'un dépôt périglaciaire reposant sur la "rasa" cantabrique près de Burela (Galice), Espagne. C.R. AC. Sc., Paris, 259 : $4092-4094$.

Feuillée P. et Rat P., 1971. - Structures et paléogéographies pyrénéocantabriques. Histoire structurale du Golfe de Gascogne, t. 2, V 1. p. 1-48.

Godwin H., Suggate R.P. et Willis E.H., 1958. Radiocarbon dating of the eustatic rise in Oceanlevel. Nature, 181: 1518-1519.

Godwin H. et Willis E.H., 1959. - Radiocarbon dating of the Late Glacial Period in Britain. Proc. Roy. Soc. (London), Ser. B, 150 : 199-215.

Grisez L., 1961. - Etudes de quelques formations littorales de l'Ouest des Asturies. Bull. Ass. Géogr. français, $\mathrm{n}^{\circ} 298$.

Guilcher A., 1955. - La plage ancienne de La Franca (Asturies). C.R. Acad. Sc. Paris, t. 241, p. 1603-1605.

Hernandez Pacheco E., 1932. - Las costas de la peninsula hispanica y sus movimientos. Assoc. Esp. para el Prog. Cienc., Madrid, p. 89-120.

Hernandez Pacheco F. et Asensio Amor I., 1959. Materiales sedimentarios sobre la rasa cantabrica. Tramo comprendido entre las rias del eo y Foz. Bol. Real. Soc. Esp. Hist. Nat., t. LVII, p. 75-100.

Hernandez Pacheco F. et Asensio Amor I., Materiales sedimentarios sobre la rasa cantabrica. II Tramo comprendido entre la ria de Foz y el casco urbano de Burela. Bol. Real. Soc. Esp. Hist. Nat., t. LVIIl, p. 73-83.

Hernandez Pacheco F. et Asensio Amor I., 1961. Materiales sedimentarios sobre la rasa catabrica. III. Tramo asturiano comprendido entre Santiago de Villapedre (Navia) y Cadavedo (Luarca). Bol. Real. Soc. Esp. Hist. Nat., t. LIX, p. 207-223.

Hernandez Pacheco F. et Asensio Amor 1., 1962. Materiales sedimentarios sobre la rasa cantabrica. IV. Tramo comprendido entre Santiago de Villapedre y el valle del rio Porcia. Bol. Real. Soc. Esp. Hist. Nat., t. 60, p. 65-76.

Hernandez Pacheco F. et Asensio Amor I., 1963. Materiales sedimentarios sobre la rasa cantabrica. V. Tramo comprendido entre el valle del rio Porcia y la ria del Eo. Bol. Real. Soc. Esp. Hist. Nat., t. 61, p. 89-120. 
Hernandez Pacheco F. et Asensio Amor I., 1964. Recientes investigaciones sobre la genesis de la rasa litoral cantabrico. Tramo final del Valle del rio Navia. Asturias. Bol. Real. Soc. Esp. Hist. Nat., t. 62, p. 61-89.

Hernandez Sampelayo P., 1913-14. - Estudio geologico de las costas de la Provincia de Lugo. B.I.G.M.E., t. $34,2^{\mathrm{e}}$ ser., p. $81-171$.

Leroi-Gouhran A., 1968. - L'abri du Facteur à Tursac (Dordogne). III. Analayse pollinique. Gallia-Préhistoire, II (I) 123-132.

Leroi-Gouhran A., 1971. - La fin du Tardiglaciaire et les industries préhistoriques (Pyrénées-Cantabres). Munibe (San Sebastian) Soc. Cien. Nat. Arauzadi, $23(2-3)$ 249-254.

Lopis Lado N., 1955-56. - Los depositos de la costa cantabrica entre los cabos Bustos y Vidio (Asturias). Speleon, t. VI, $\mathrm{n}^{\circ} 4$ p. 333-347.

Lopis Llado N., 1964. - Estudio geologico de los alrededores de Luarca (Asturias). Excma diputacion proviricial de Oyiedo. Instituto de Estudios Asturianos. Instituto geologico y mineros de Espana.

Mary G., 1967. - Les niveaux marins fossiles de la région de Otur (Luarca Asturies, Espagne). Bull. Soc. Lin. de Normandie, 10 e sér., $8^{\mathrm{e}}$ vol., p. 38-52.

Mary G., 1968. - Les formations marines actuelles et fossiles à l'embouchure du rio Esba (Luarca, Asturies, Espagne). Cah. Océan, XX, 8, p. 683693.

Mary G., 1970. - La rasa cantabrique entre Luarca et Ribadeo (Asturies, Espagne). Rev. Géol. Astur., Ano XIV, num. 4, p. 45-48.

Mary G., 1971. - Les formations quaternaires de la côte asturienne (Espagne) entre Ribadesella et Comillas. Bull. AFEQ, 2, p. 11-118.

Mary G., 1971. - Les hautes surfaces d'abrasion marine de la côte asturienne (Espagne). Histoire structurale du Golfe de Gascogne, t. 2, V5, p. 1-12. I.F.P. Technip.

Mary G., 1972. - Le Quaternaire du Cap de Esbarradoïro (Luarca, Asturies, Espagne). Bre. Géol. Asturica, ano XVI, $\mathrm{n}^{\circ} 4$, p. 53-56.

Mary G., Medus J., 1971. - Présence de Sparnacien à la base d'une "rasa" au Monte Granda à l'Ouest de Aviles (Asturies, Espagne). C.R.S. S.G.F., fasc. 17, p. 125 .

Mary G., Beaulieu J.L. de et Médus J., 1973. - Un diagramme sporopollinique et des datations $14 \mathrm{C}$ pour la tourbière du Llano Ronanzas (AsturiesEspagne). B.S.G.F., Paris, 7, 15 (I).
Morzadec Kerfourn M.Th., 1969. - Variations de la ligne du rivage au cours du Post-Glaciaire le long de la côte nord du Finistère. Analyses polliniques de tourbes et de dépôts organiques littoraux. Bull. A.F.E.Q., 4, p. 285-318.

Nonn H., 1966. - Les régions côtières de la Galice (Espagne). Etude géomorphologique. Publ. Fac. Lettres de l'Université de Strasbourg. Fondation Baulig, III.

Oldfield F., 1960. - The coastal mud-bed at Mouligna, Bidart, and the age of the asturian industry in the Pays basque. Pollen et Spores, 2 (I) $57-70$.

Oldfield F., 1964. - Late Quaternary vegetational history in South west France, Pollen et Spores, 6 (I) $157-168$.

Paquereau M.M., 1969. - Etude palynologique du Würm I du Pech de l'Aze (Dordogne). Quaternaire $X I$, Roma, 227-235.

Paquereau M.M., 1970. - Flores et Climats paléolithiques dans le Sud-Ouest de la France. Rev. Géogr. Phys. Géol. Dynam., (2) 12 (2) 109-116.

Ters M., 1973. - Les variations du niveau marin depuis 10000 ans le long du littoral atlantique français. Suppl. Bull. $A F E Q$ n $^{\circ} 36$, p. 114-136.

Van Campo M. et Aymonin G., 1962. - Le problème de l'histoire de la flore et de la végétation dans les Cévennes méridionales vu sous l'angle de l'analyse pollinique. Flora, 152 : 679-688.

Van Campo M., 1969. - Végétation würmienne en France. Données bibliographiques. Hypothèses Etudes Françaises sur le Quaternaire. $8^{e}$ Congr. Inter. I.N.Q.U.A., Paris 1969. Suppl. Bull. A.F.E.Q., 104-111.

Vigneaux M., 1973. - Interprétation comparée de données climatiques. stratigraphiques et paléogéographiques: Applications au Quaternaire récent du Plateau continental au S.W. de Rochebonne (Golfe de Gascogne). in : Les Méthodes Quantitatives d'Etude des Variations de climat au cours du pleistocène. Coll. Intern. C.N.R.S., $n^{\circ} 219$, Gif sur Yvette, 4 pp.

Virgili G., Mary G., Suarez Vega L.C., Breil J.M., 1968. - Depositos marinos del Cuaternario antigo (?) sobre la cuarcita armoricana del Cabo Torres (Gijon). Brev. Asturica, ano XII, $n^{\circ} 2$, p. 4-8. 